\title{
Wigner-Weyl isomorphism for quantum mechanics on Lie groups
}

\author{
N. Mukunda ${ }^{a}$ \\ Centre for Theoretical Studies, Indian Institute of Science, Bangalore 560 012, India \\ G. Marmo ${ }^{\text {b) }}$ and A. Zampinic) \\ Dipartimento di Scienze Fisiche, Universita di Napoli Federico II and INFN, \\ Via Cinzia, 80126 Napoli, Italy \\ S. Chaturvedi ${ }^{d)}$ \\ School of Physics, University of Hyderabad, Hyderabad 500 046, India \\ R. Simon ${ }^{\mathrm{e}}$ \\ The Institute of Mathematical Sciences, C. I. T. Campus, Chennai 600 113, India
}

\begin{abstract}
The Wigner-Weyl isomorphism for quantum mechanics on a compact simple Lie group $G$ is developed in detail. Several features are shown to arise which have no counterparts in the familiar Cartesian case. Notable among these is the notion of a semiquantized phase space, a structure on which the Weyl symbols of operators turn out to be naturally defined and, figuratively speaking, located midway between the classical phase space $T^{*} G$ and the Hilbert space of square integrable functions on $G$. General expressions for the star product for Weyl symbols are presented and explicitly worked out for the angle-angular momentum case.
\end{abstract}

\section{INTRODUCTION}

It is well known that the method of Wigner distributions, ${ }^{1}$ which describes every state of a quantum mechanical system by a corresponding real quasiprobability density on the classical phase space, is dual to the Weyl mapping ${ }^{2}$ of classical dynamical variables to quantum mechanical operators. Together they provide the Wigner-Weyl isomorphism, whereby both states and operators in quantum mechanics can be given $c$-number descriptions on the classical phase space. The trace of the product of two operators is then calculable as the integral of the product of the two corresponding Weyl symbols or phase space functions. Combined with the work of Moyal, ${ }^{3}$ which shows how products and commutators of operators are expressed in phase space language, this entire development may be called the Wigner-Weyl-Moyal or WWM method in quantum mechanics and has been instrumental in giving rise to the fertile subject of deformation quantization. ${ }^{4}$ An important feature of the Wigner distribution is that while it is not by itself a phase space probability density, its marginals obtained by, respectively, integrating over momenta or over coordinates do reproduce the quantum mechanical expressions for probability densities in coordinate and in momentum space, respectively.

The WWM method has been studied most extensively in the case of Cartesian systems in quantum mechanics. By this we mean those systems whose configuration space $Q$ is $\mathbb{R}^{n}$ for some integer $n \geqslant 1$. The classical phase space is then $T^{*} Q \simeq \mathbb{R}^{2 n}$. While Schrödinger wave functions are

\footnotetext{
${ }^{a)}$ Electronic mail: nmukunda@cts.iisc.ernet.in

${ }^{b}$ Electronic mail: giuseppe.marmo@na.infn.it

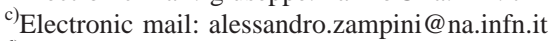

${ }^{\mathrm{d})}$ Electronic mail: scsp@uohyd.ernet.in

${ }^{\mathrm{e})}$ Electronic mail: simon@imsc.res.in
} 
square integrable functions on $\mathbb{R}^{n}$, both Wigner distributions and Weyl symbols are functions on $\mathbb{R}^{2 n}$. Quantum kinematics can be expressed via the Heisenberg canonical commutation relations for Cartesian coordinates and their conjugate momenta, or via the exponentiated Weyl form using families of unitary operators. An important feature in this case is that as far as their eigenvalue spectra are concerned, the momenta do not experience any quantization on their own; they account for the second factor in $T^{*} Q \simeq \mathbb{R}^{2 n} \simeq \mathbb{R}^{n} \times \mathbb{R}^{n}$. Furthermore we have in this case the Stone-von Neumann theorem on the uniqueness of the irreducible representation of the Heisenberg commutation relations, and the important roles of the groups $\operatorname{Sp}(2 n, R)$ and $\mathrm{Mp}(2 n)$ corresponding to linear canonical transformations on coordinates and momenta.

There has been for some time considerable interest in developing the Wigner-Weyl isomorphism for other kinds of quantum systems, that is, for non-Cartesian systems. ${ }^{5-16}$ In these cases, typically the underlying quantum kinematics cannot be expressed by Heisenberg-type commutation relations. The situations studied include the quantum mechanics of an angle-angular momentum pair, where the configuration space is $Q=\mathrm{S}^{1},{ }^{17,18}$ and finite state quantum systems corresponding to a finite dimensional Hilbert space. ${ }^{19,20}$ More recently, the method of Wigner distributions has been developed for quantum systems whose configuration space is a compact simple Lie group; and in the discrete case when it is a finite group of odd order. ${ }^{21,22}$ In all these departures from the Cartesian situation, an important aspect is the occurrence of new features which do not show up at all with Cartesian variables.

The aim of the present work is to develop in detail the Wigner-Weyl isomorphism for quantum mechanics on a compact simple Lie group. Here the configuration space $Q$ is a (compact simple) Lie group $G$, so the corresponding classical phase space is $T^{*} G \simeq G \times G^{*}$, where $G^{*}$ is the dual to the Lie algebra $G$ of $G$. In the quantum situation, Schrödinger wave functions are complex square integrable functions on $G$, and observables or dynamical variables are linear Hermitian operators acting on such functions. The replacements for the canonical Heisenberg commutation relations are best formulated using the (commutative) algebra of suitable smooth functions on $G$, and (say) the left regular representation of $G$ acting on functions on itself. The natural question that arises in trying to set up a Wigner-Weyl isomorphism in this case is whether quantum states and operators are to be described using suitable functions on the classical phase space $T^{*} G$. In Ref. 21 an overcomplete Wigner distribution formalism for quantum states, which transforms in a reasonable way under left and right group actions and also reproduces the natural marginal probability distributions, has been developed. The methods developed there are here exploited to set up a Wigner-Weyl isomorphism in full detail, disclosing many interesting differences compared to the Cartesian case. In particular we find that this isomorphism does not directly utilize $c$-number functions on $T^{*} G$ at all, but instead uses a combination of functions on $G$ and operators on a simpler Hilbert space, standing in a sense midway between $T^{*} G$ and the Hilbert space of the quantum system. This feature is traceable to the non-Abelian nature of $G$, something which is absent in the Cartesian case when $Q$ is the Abelian group $\mathbb{R}^{n}$.

The material of this paper is organized as follows. In Sec. II we briefly recapitulate key features of the Wigner-Weyl isomorphism for the Cartesian and angle-angular momentum cases. This sets the stage for Sec. III where we develop the quantum kinematics for situations where the configuration space is a compact Lie group and thus go beyond the Abelian cases discussed in Sec. I. This analysis leads to a proper identification of the analogues of the momenta of the Cartesian case and helps set up the Wigner distribution for such situations possessing properties expected of a Wigner distribution. The Wigner distributions so defined have a certain degree of overcompleteness about them, a circumstance forced by the non-Abelian nature of the underlying group $G$. A key ingredient in this construction is the notion of the midpoint of two group elements introduced in an earlier work. ${ }^{21}$ In Sec. IV a more compact description in terms of Weyl symbols devoid of any redundances is developed and correspondences facilitating transition from the Cartesian case to more general situations are established. The results of Sec. IV are exploited in Sec. V towards defining a star product between Weyl symbols for operators and the general expression for the star product is explicitly worked out for the non-Cartesian, albeit Abelian case of angle-angular momentum. Section VI is devoted to analyzing the minimal structure on which the Weyl symbols for 
operators find their natural definition. This leads to the concept of a noncommutative cotangent space or a semiquantized phase space the ramifications of which are examined further towards highlighting the structural similarity between classical phase space functions and the Weyl symbols. A short appendix contains some technical details concerning results used in Sec. V.

\section{THE WIGNER-WEYL ISOMORPHISM: CARTESIAN AND ANGLE-ANGULAR MOMENTUM CASES}

In this section we recall briefly the relevant structures needed to set up the Wigner-Weyl isomorphism for Cartesian quantum mechanics. This is to facilitate comparison with the Lie group case later on. For simplicity we choose one degree of freedom only, as the extension to $Q=\mathbb{R}^{n}$ is straightforward. We also recall the angle-angular momentum case, $Q=\mathrm{S}^{1}$, where we already see significant differences from the Cartesian case; these increase when we go to $Q=G$.

One-dimensional Cartesian quantum mechanics: The canonical Heisenberg commutation relation between Hermitian coordinate and momentum operators $\hat{q}$ and $\hat{p}$, fixing the kinematics, is

$$
[\hat{q}, \hat{p}]=i .
$$

In the unitary Weyl form this is expressed as follows:

$$
\begin{aligned}
& U(p)=\exp (i p \hat{q}), \quad V(q)=\exp (-i q \hat{p}), \\
& U(p) V(q)=V(q) U(p) e^{i q p}, \quad q, p \in \mathbb{R} .
\end{aligned}
$$

In the Cartesian case the exponentials can be combined to define a phase space displacement operator

$$
D(q, p)=U(p) V(q) e^{-i q p / 2}=V(q) U(p) e^{i q p / 2}=\exp (i p \hat{q}-i q \hat{p}) .
$$

However this cannot be done even in the single angle-angular momentum pair case, and also when we treat the Lie group case. We therefore use expressions in which the exponentials are kept separate.

The standard form of the unique irreducible representation of Eqs. (2.1) and (2.2) uses the Hilbert space of square integrable functions $\psi(q)$ on R. Introducing as usual an ideal basis of eigenvectors of $\hat{q}$ we have

$$
\begin{gathered}
\mathcal{H}=L^{2}(\mathbb{R})=\left\{\left.\psi(q)\left|\|\psi\|^{2}=\int_{\mathbb{R}} \mathrm{d} q\right| \psi(q)\right|^{2}<\infty\right\}, \\
\psi(q)=\langle q \mid \psi\rangle, \quad \hat{q}|q\rangle=q|q\rangle, \\
\left\langle q^{\prime} \mid q\right\rangle=\delta\left(q^{\prime}-q\right) .
\end{gathered}
$$

On such $\psi(q)$ (subject to relevant domain conditions) the actions of $\hat{q}, \hat{p}, U(p), V(q)$ are

$$
\begin{gathered}
(\hat{q} \psi)(q)=q \psi(q), \quad(\hat{p} \psi)(q)=-i \frac{\mathrm{d}}{\mathrm{d} q} \psi(q), \\
\left(U\left(p^{\prime}\right) \psi\right)(q)=e^{i p^{\prime} q} \psi(q), \quad\left(V\left(q^{\prime}\right) \psi\right)(q)=\psi\left(q-q^{\prime}\right) .
\end{gathered}
$$

The momentum space description of $|\psi\rangle$ uses the Fourier transform of $\psi(q)$; in terms of the ideal eigenstates $|p\rangle$ for $\hat{p}$,

$$
\widetilde{\psi}(p)=\langle p \mid \psi\rangle=\int_{\mathbb{R}} \frac{\mathrm{d} q}{\sqrt{2 \pi}} \exp (-i p q) \psi(q),
$$




$$
\|\psi\|^{2}=\int_{\mathbb{R}} \mathrm{d} p|\widetilde{\psi}(p)|^{2}
$$

The displacement operators (2.2) form a complete trace orthonormal set (in the continuum sense) in the space of operators on $\mathcal{H}$,

$$
\operatorname{Tr}\left(\left(U\left(p^{\prime}\right) V\left(q^{\prime}\right)\right)^{\dagger} U(p) V(q)\right)=2 \pi \delta\left(q^{\prime}-q\right) \delta\left(p^{\prime}-p\right) .
$$

The completeness property will be used later.

The definitions of the Wigner distribution for a normalized pure state $|\psi\rangle \in \mathcal{H}$, or more generally for a mixed state with density operator $\hat{\rho}$, are

$$
\begin{gathered}
W(q, p)=\frac{1}{2 \pi} \int_{\mathrm{R}} \mathrm{d} q^{\prime} \psi\left(q-\frac{1}{2} q^{\prime}\right) \psi\left(q+\frac{1}{2} q^{\prime}\right)^{*} \exp \left(i p q^{\prime}\right) \\
W(q, p)=\frac{1}{2 \pi} \int_{\mathbb{R}} \mathrm{d} q^{\prime}\left\langle q-\frac{1}{2} q^{\prime}|\hat{\rho}| q+\frac{1}{2} q^{\prime}\right\rangle \exp \left(i p q^{\prime}\right)
\end{gathered}
$$

(The dependences on $|\psi\rangle, \hat{\rho}$ are left implicit.) While $W(q, p)$ is real though not always nonnegative, the recovery of the marginal position and momentum space probability densities is assured by

$$
\int_{\mathbb{R}} \mathrm{d} p W(q, p)=\langle q|\hat{\rho}| q\rangle, \quad \int_{\mathbb{R}} \mathrm{d} q W(q, p)=\langle p|\hat{\rho}| p\rangle
$$

It is possible to express $W(q, p)$ in a more compact form by introducing a family of Hermitian operators $\hat{W}(q, p)$ on $\mathcal{H}$ with interesting algebraic properties. They are essentially the double Fourier transforms of the displacement operators (2.2),

$$
\begin{gathered}
W(q, p)=\operatorname{Tr}(\hat{\rho} \hat{W}(q, p)), \\
\hat{W}(q, p)=\hat{W}(q, p)^{\dagger}=\frac{1}{(2 \pi)^{2}} \int_{\mathbb{R}} \int_{\mathbb{R}} \mathrm{d} q^{\prime} \mathrm{d} p^{\prime} U\left(p^{\prime}\right) V\left(q^{\prime}\right) e^{i p q^{\prime}-i p^{\prime}\left(q+(1 / 2) q^{\prime}\right)} .
\end{gathered}
$$

It has been shown in Ref. 17 that, apart from sharing the trace orthonormality property (2.7) which is preserved by the Fourier transformation,

$$
\operatorname{Tr}\left(\hat{W}\left(q^{\prime}, p^{\prime}\right) \hat{W}(q, p)\right)=\frac{1}{2 \pi} \delta\left(q^{\prime}-q\right) \delta\left(p^{\prime}-p\right),
$$

we have the following behaviors under anticommutation with $\hat{q}$ and $\hat{p}$ :

$$
\frac{1}{2}\{\hat{q}, \hat{W}(q, p)\}=q \hat{W}(q, p), \quad \frac{1}{2}\{\hat{p}, \hat{W}(q, p)\}=p \hat{W}(q, p) .
$$

Thus we may regard $\hat{W}(q, p)$ as operator analogues of Dirac delta functions concentrated at individual phase space points. In Ref. 19 they have been called phase point operators.

Turning to the Weyl map, it takes a general classical dynamical variable, a (square integrable) function $a(q, p)$ on the classical phase space, to a corresponding (Hilbert-Schmidt) operator $\hat{A}$ on $\mathcal{H}$ : 


$$
\begin{aligned}
a(q, p) & \rightarrow \widetilde{a}\left(p^{\prime}, q^{\prime}\right)=\int_{\mathbb{R}} \int_{\mathbb{R}} \mathrm{d} q \mathrm{~d} p a(q, p) e^{i\left(p q^{\prime}-q p^{\prime}\right)} \\
& \rightarrow \hat{A}=\frac{1}{2 \pi} \int_{\mathbb{R}} \int_{\mathbb{R}} \mathrm{d} q^{\prime} \mathrm{d} p^{\prime} \widetilde{a}\left(p^{\prime}, q^{\prime}\right) U\left(p^{\prime}\right) V\left(q^{\prime}\right) e^{-i q^{\prime} p^{\prime} / 2} .
\end{aligned}
$$

The important property of this map is that traces of operators on $\mathcal{H}$ go into integrals over phase space,

$$
\operatorname{Tr}\left(\hat{A}^{\dagger} \hat{B}\right)=\int_{\mathbb{R}} \int_{\mathbb{R}} \mathrm{d} q \mathrm{~d} p a(q, p) * b(q, p) .
$$

One can immediately see that the relation between $a(q, p)$ and $\hat{A}$ is given by

$$
\hat{A}=2 \pi \int_{\mathbb{R}} \int_{\mathbb{R}} \mathrm{d} q \mathrm{~d} p a(q, p) \hat{W}(q, p),
$$

thus establishing that the Wigner and Weyl maps are inverses of one another. Indeed extending the definition of the Wigner distribution (2.10) to a general operator $\hat{A}$ on $\mathcal{H}$, we have

$$
a(q, p)=\operatorname{Tr}(\hat{A} \hat{W}(q, p)) .
$$

It is this kind of isomorphism that we wish to develop when $\mathbb{R}$ is replaced by a compact simple Lie group $G$.

The angle-angular momentum case: We now trace the changes which appear if we replace the Cartesian variable $q \in \mathbb{R}$ by an angle $\theta \in(-\pi, \pi)$. The corresponding Hermitian operator is denoted by $\hat{\theta}$, with eigenvalues $\theta$; its canonical conjugate $\hat{M}$ has integer eigenvalues $m$ $=0, \pm 1, \pm 2, \ldots$. Thus $m \in \mathbb{Z}$ unlike the Cartesian $p$, so $\hat{M}$ is already quantized. The replacements for Eqs. (2.4) and (2.6) are

$$
\begin{gathered}
\mathcal{H}=L^{2}\left(\mathrm{~S}^{1}\right)=\left\{\left.\psi(\theta)\left|\|\psi\|^{2}=\int_{-\pi}^{\pi} \mathrm{d} \theta\right| \psi(\theta)\right|^{2}<\infty\right\}, \\
\psi(\theta)=\langle\theta \mid \psi\rangle, \quad\left\langle\theta^{\prime} \mid \theta\right\rangle=\delta\left(\theta^{\prime}-\theta\right), \quad \hat{\theta}|\theta\rangle=\theta|\theta\rangle, \\
\psi_{m}=\langle m \mid \psi\rangle=\frac{1}{2 \pi} \int_{-\pi}^{\pi} \mathrm{d} \theta e^{-i m \theta} \psi(\theta), \\
\|\psi\|^{2}=\sum_{m \in \mathbb{Z}}\left|\psi_{m}\right|^{2}, \\
\hat{M}|m\rangle=m|m\rangle, \quad\langle\theta \mid m\rangle=\frac{1}{\sqrt{2 \pi}} e^{i m \theta} .
\end{gathered}
$$

In place of the Heisenberg commutation relation (2.1), we have only the exponentiated Weyl version,

$$
\begin{gathered}
U(m)=\exp (i m \hat{\theta}), \quad V(\theta)=\exp (-i \theta \hat{M}), \\
U(m) V(\theta)=V(\theta) U(m) e^{i m \theta} .
\end{gathered}
$$


With the actions

$$
\begin{aligned}
& \left(U\left(m^{\prime}\right) \psi\right)(\theta)=e^{i m^{\prime} \theta} \psi(\theta), \\
& \left(V\left(\theta^{\prime}\right) \psi\right)(\theta)=\psi\left(\left[\theta-\theta^{\prime}\right]\right), \\
& {\left[\theta-\theta^{\prime}\right]=\theta-\theta^{\prime} \bmod 2 \pi,}
\end{aligned}
$$

we have an irreducible system on $\mathcal{H}=L^{2}\left(\mathrm{~S}^{1}\right)$. The analogues of the displacement operators (2.3) are now

$$
U(m) V(\theta) e^{-i m \theta / 2}=V(\theta) U(m) e^{i m \theta / 2},
$$

but here the exponents cannot be combined. They do however form a complete trace orthonormal system,

$$
\operatorname{Tr}\left(\left(U\left(m^{\prime}\right) V\left(\theta^{\prime}\right)\right)^{\dagger} U(m) V(\theta)\right)=2 \pi \delta_{m m^{\prime}} \delta\left(\theta^{\prime}-\theta\right) .
$$

With this preparation we can turn to the definition of the Wigner distribution and the Weyl map. For a given density operator $\hat{\rho}$ on $\mathcal{H}$, the former is

$$
W(\theta, m)=\frac{1}{2 \pi} \int_{-\pi}^{\pi} \mathrm{d} \theta^{\prime}\left\langle\theta-\frac{1}{2} \theta^{\prime}|\hat{\rho}| \theta+\frac{1}{2} \theta^{\prime}\right\rangle \exp \left(i m \theta^{\prime}\right) .
$$

We see immediately that this is not a function on the classical phase space $T^{*} \mathrm{~S}^{1} \simeq \mathrm{S}^{1} \times \mathbb{R}$, which is a cylinder, but on a partially quantized space $S^{1} \times \mathbb{Z}$. We may regard this space as standing somewhere in between $T^{*} \mathrm{~S}^{1}$ and the fully quantum mechanical Hilbert space and operator setup. The marginals are properly reproduced in the sense that

$$
\begin{gathered}
\int_{-\pi}^{\pi} \mathrm{d} \theta W(\theta, m)=\langle m|\hat{\rho}| m\rangle, \\
\sum_{m \in Z} W(\theta, m)=\langle\theta|\hat{\rho}| \theta\rangle .
\end{gathered}
$$

We can display $W(\theta, m)$ as

$$
\begin{gathered}
W(\theta, m)=\operatorname{Tr}(\hat{\rho} \hat{W}(\theta, m)), \\
\hat{W}(\theta, m)=\hat{W}(\theta, m)^{\dagger}=\frac{1}{(2 \pi)^{2}} \sum_{m^{\prime} \in \mathbb{Z}} \int_{-\pi}^{\pi} \mathrm{d} \theta^{\prime} U\left(m^{\prime}\right) V\left(\theta^{\prime}\right) e^{i m \theta^{\prime}-i m^{\prime}\left(\theta+1 / 2 \theta^{\prime}\right),}
\end{gathered}
$$

and like their Cartesian counterparts these operators form a trace orthonormal system,

$$
\operatorname{Tr}\left(\hat{W}\left(\theta^{\prime}, m^{\prime}\right) \hat{W}(\theta, m)\right)=\frac{1}{2 \pi} \delta\left(\theta^{\prime}-\theta\right) \delta_{m m^{\prime}} .
$$

In a similar spirit, the Weyl map now takes any classical function $a(\theta, m)$ on $\mathrm{S}^{1} \times \mathbb{Z}$ into an operator on $L^{2}\left(\mathrm{~S}^{1}\right)$, 


$$
\begin{aligned}
a(\theta, m) & \rightarrow \widetilde{a}\left(m^{\prime}, \theta^{\prime}\right)=\sum_{m \in Z} \int_{-\pi}^{\pi} \mathrm{d} \theta a(\theta, m) e^{i\left(m \theta^{\prime}-m^{\prime} \theta\right)} \\
& \rightarrow \hat{A}=\frac{1}{2 \pi} \sum_{m^{\prime} \in \mathbb{Z}} \int_{-\pi}^{\pi} \mathrm{d} \theta^{\prime} \widetilde{a}\left(m^{\prime}, \theta^{\prime}\right) U\left(m^{\prime}\right) V\left(\theta^{\prime}\right) e^{-i m^{\prime} \theta^{\prime} / 2} .
\end{aligned}
$$

Then the trace operation becomes, as in (2.14),

$$
\operatorname{Tr}\left(\hat{A}^{\dagger} \hat{B}\right)=\sum_{m \in Z} \int_{-\pi}^{\pi} \mathrm{d} \theta a(\theta, m) * b(\theta, m) .
$$

Combining Eqs. (2.24) and (2.26) we are able to get the analogue to (2.15),

$$
\hat{A}=2 \pi \sum_{m \in Z} \int_{-\pi}^{\pi} \mathrm{d} \theta a(\theta, m) \hat{W}(\theta, m) .
$$

In this way the similarities as well as important differences compared to the Cartesian case are easily seen.

\section{QUANTUM KINEMATICS IN THE LIE GROUP CASE AND THE WIGNER DISTRIBUTION}

Let $G$ be a (non-Abelian) compact simple Lie group of order $n$, with elements $g, g^{\prime}, \ldots$ and composition law $g^{\prime}, g \rightarrow g^{\prime} g$. To set up the kinematics appropriate for a quantum system with configuration space $Q=G$, it is simplest to begin with the Hilbert space of Schrödinger wave functions. The normalized left and right invariant volume element on $G$ is written as $\mathrm{d} g$. For suitable functions $f(g)$ on $G$ we have the invariances and normalization condition

$$
\begin{gathered}
\int_{G} \mathrm{~d} g f(g)=\int_{G} \mathrm{~d} g\left(f\left(g^{\prime} g\right) \quad \text { or } f\left(g g^{\prime}\right) \text { or } f\left(g^{-1}\right)\right), \\
\int_{G} \mathrm{~d} g=1 .
\end{gathered}
$$

Correspondingly we can introduce a Dirac delta function on $G$ characterized by

$$
\int_{G} \mathrm{~d} g\left(\delta\left(g^{\prime-1} g\right) \quad \text { or } \delta\left(g g^{\prime-1}\right) \quad \text { or } \delta\left(g^{-1} g^{\prime}\right) \quad \text { or } \delta\left(g^{\prime} g^{-1}\right)\right) f(g)=f\left(g^{\prime}\right)
$$

Thus $\delta(g)$ is a delta function concentrated at the identity element $e \in G$.

We take the Hilbert space $\mathcal{H}$ for the quantum system to be made up of all complex square integrable functions on $G$ :

$$
\mathcal{H}=L^{2}(G)=\left\{\left.\psi(g) \in C\left|\|\psi\|^{2}=\int_{G} \mathrm{~d} g\right| \psi(g)\right|^{2}<\infty\right\} .
$$

A convenient basis of ideal vectors $|g\rangle$ can be introduced such that for a general $|\psi\rangle \in \mathcal{H}$ we may write

$$
\psi(g)=\langle g \mid \psi\rangle, \quad\left\langle g^{\prime} \mid g\right\rangle=\delta\left(g^{\prime} g^{-1}\right) .
$$

The notion of position coordinates is intrinsically captured by the commutative algebra representing real valued smooth functions $f(g)$ on $G$, i.e., $f \in \mathcal{F}(G)$. To each such function we associate a Hermitian multiplicative operator $\hat{f}$ on $\mathcal{H}$ : 


$$
\begin{gathered}
f \in \mathcal{F}(G) \rightarrow \hat{f}=\int_{G} \mathrm{~d} g f(g)|g\rangle\langle g|, \\
(\hat{f} \psi)(g)=f(g) \psi(g) .
\end{gathered}
$$

Thus all these operators commute with one another, being diagonal in the position description $\psi(g)$ of $|\psi\rangle$.

To complete the kinematics and to obtain an irreducible system of operators on $\mathcal{H}$ we have to adjoin suitable momenta. Here we have two choices, corresponding to the left and right translations of $G$ on itself by group action. We choose the former, and so define a family of unitary operators $V(g)$ to give the left regular representation of $G$ :

$$
\begin{gathered}
\left(V\left(g^{\prime}\right) \psi\right)(g)=\psi\left(g^{\prime-1} g\right), \\
V\left(g^{\prime}\right)|g\rangle=\left|g^{\prime} g\right\rangle .
\end{gathered}
$$

They obey

$$
\begin{gathered}
V\left(g^{\prime}\right) V(g)=V\left(g^{\prime} g\right), \\
V(g)^{\dagger} V(g)=I .
\end{gathered}
$$

To identify their Hermitian generators, we introduce a basis $\left\{e_{r}\right\}$ in the Lie algebra $\underline{G}$ of $G$. Using the exponential map $\underline{G} \rightarrow G$, we write a general $g \in G$ as

$$
g=\exp \left(\alpha^{r} e_{r}\right)
$$

the sum on $r$ being from 1 to $n$. The generators $\hat{J}_{r}$ of $V(g)$ are then identified by

$$
V\left(\exp \left(\alpha^{r} e_{r}\right)\right)=\exp \left(-i \alpha^{r} \hat{J}_{r}\right) .
$$

These are Hermitian operators on the Hilbert space $\mathcal{H}$, obeying commutation relations involving the structure constants $C_{r s}^{t}$ of $G$ :

$$
\left[\hat{J}_{r}, \hat{J}_{s}\right]=i C_{r s}{ }^{t} \hat{J}_{t} .
$$

On Schrödinger wave functions $\psi(g)$ each $\hat{J}_{r}$ acts as a first order partial differential operator; indeed if the (right invariant) vector fields generating the left action of $G$ on itself are written as $X_{r}$, then we have

$$
\hat{J}_{r} \psi(g)=i X_{r} \psi(g)
$$

The commutation relations (3.10) are direct consequences of similar commutation relations among the vector fields $X_{r}$.

The analogue of the Cartesian Heisenberg-Weyl system (2.1) and (2.2) is now obtained by setting together the following ingredients:

$$
\begin{gathered}
f_{1}, f_{2} \in \mathcal{F}(G) \rightarrow \hat{f}_{1} \hat{f}_{2}=\hat{f}_{2} \hat{f}_{1}, \\
f \in \mathcal{F}(G), \quad g^{\prime} \in G \rightarrow V\left(g^{\prime}\right) \hat{f} V\left(g^{\prime}\right)^{-1}=\hat{f}^{\prime},
\end{gathered}
$$




$$
f^{\prime}(g)=f\left(g^{\prime-1} g\right),
$$

along with the representation property (3.7) for $V(g)$. This is in the spirit of the unitary Weyl system (2.2). In infinitesimal terms we have

$$
\left[\hat{J}_{r}, \hat{f}\right]=i \widehat{\left(X_{r} f\right)}
$$

combined with (3.10). The space $\mathcal{H}$ is indeed irreducible with respect to the family of operators $\{\hat{f}, V(\cdot)\}$ or equivalently $\left\{\hat{f}, \hat{J}_{r}\right\}$.

We can express functions of position also via unitary operators in the Weyl spirit as follows: for each real $f \in \mathcal{F}(G)$, we define the unitary operator $U(f)$ by

$$
U(f)=e^{i \hat{f}}:(U(f) \psi)(g)=e^{i f(g)} \psi(g) .
$$

It is then easy to see that we have the relations

$$
\begin{gathered}
\left(U(f) V\left(g^{\prime}\right) \psi\right)(g)=e^{i f(g)} \psi\left(g^{\prime-1} g\right), \\
\left(V\left(g^{\prime}\right) U(f) \psi\right)(g)=e^{i f\left(g^{\prime-1} g\right)} \psi\left(g^{\prime-1} g\right), \\
\left(U(f) V\left(g^{\prime}\right)\left(V\left(g^{\prime}\right) U(f)\right)^{\dagger} \psi\right)(g)=e^{i f(g)-i f\left(g^{\prime-1} g\right)} \psi(g),
\end{gathered}
$$

which is in the spirit of Eqs. (2.2) and (2.18), except that $f$ is not restricted to be linear in any coordinate variables.

We see here that unlike in the $n$-dimensional Cartesian case the canonical momenta are a noncommutative system. Therefore the analogue or generalization of the single momentum eigenstate $|\underline{p}\rangle$ in the Cartesian situation will turn out to be a generally multidimensional Hermitian irreducible representation of (3.10), namely the generators of some unitary irreducible representation (UIR) of $G$. We will see this in detail as we proceed.

For completeness we should mention the operators giving the right regular representation of $G$. These are, say, $\widetilde{V}(g)$, defined by and obeying

$$
\begin{gathered}
\left(\widetilde{V}\left(g^{\prime}\right) \psi\right)(g)=\psi\left(g g^{\prime}\right), \\
\widetilde{V}\left(g^{\prime}\right)|g\rangle=\left|g g^{\prime-1}\right\rangle, \\
\widetilde{V}\left(g^{\prime}\right) \widetilde{V}(g)=\widetilde{V}\left(g^{\prime} g\right), \\
V\left(g^{\prime}\right) \widetilde{V}(g)=\widetilde{V}(g) V\left(g^{\prime}\right) .
\end{gathered}
$$

However as is well known their generators $\hat{\widetilde{J}}_{r}$ are determined by $\hat{J}_{r}$ and the matrices $\left(\mathcal{D}_{r}^{s}(g)\right)$ of the adjoint representation of $G$, by

$$
\hat{\widetilde{J}}_{r}=-\mathcal{D}_{r}^{s}(g) \hat{J}_{s} .
$$

Therefore it suffices to regard the collection of operators $\{\hat{f}, V(\cdot)\}$ as providing the replacement for the Heisenberg-Weyl system in the present case.

Complementary to the position basis $|g\rangle$ for $\mathcal{H}$ is a momentum basis. This can be set up using the Peter-Weyl theorem involving all the UIR's of $G$. We denote the various UIR's by $j$, with dimension $N_{j}$; we label rows and columns within the $j$ th UIR by magnetic quantum numbers $m, n$. Thus the unitary matrix representing $g \in G$ in the $j$ th UIR is 


$$
g \rightarrow\left(D_{m n}^{j}(g)\right) .
$$

In general each of $j, m, n$ is a collection of several independent discrete quantum numbers, and there is a freedom of unitary changes in the choice of $m, n$. In addition to unitarity and the composition law,

$$
\begin{gathered}
\sum_{n} D_{m n}^{j}(g) * D_{m^{\prime} n}^{j}(g)=\delta_{m m^{\prime}}, \\
\sum_{n} D_{m n}^{j}\left(g^{\prime}\right) D_{n n^{\prime}}^{j}(g)=D_{m n^{\prime}}^{j}\left(g^{\prime} g\right),
\end{gathered}
$$

we have orthogonality and completeness properties,

$$
\begin{gathered}
\int_{G} \mathrm{~d} g D_{m n}^{j}(g) D_{m^{\prime} n^{\prime}}^{j^{\prime}}(g) *=\delta_{j j^{\prime}} \delta_{m m^{\prime}} \delta_{n n^{\prime}} / N_{j}, \\
\sum_{j m n} N_{j} D_{m n}^{j}(g) D_{m n}^{j}\left(g^{\prime}\right) *=\delta\left(g^{-1} g^{\prime}\right) .
\end{gathered}
$$

Then a simultaneous complete reduction of both representations $V(\cdot), \widetilde{V}(\cdot)$ of $G$ is achieved by passing to a new orthonormal basis $|j m n\rangle$ for $\mathcal{H}$. Its definition and basic properties are

$$
\begin{gathered}
|j m n\rangle=N_{j}^{1 / 2} \int_{G} \mathrm{~d} g D_{m n}^{j}(g)|g\rangle, \\
\left\langle j^{\prime} m^{\prime} n^{\prime} \mid j m n\right\rangle=\delta_{j^{\prime} j} \delta_{m^{\prime} m} \delta_{n^{\prime} n}, \\
V(g)|j m n\rangle=\sum_{m^{\prime}} D_{m m^{\prime}}^{j}\left(g^{-1}\right)\left|j m^{\prime} n\right\rangle, \\
\widetilde{V}(g)|j m n\rangle=\sum_{n^{\prime}} D_{n^{\prime} n}^{j}(g)\left|j m n^{\prime}\right\rangle .
\end{gathered}
$$

Therefore in $|j m n\rangle$ the index $n$ counts the multiplicity of occurrence of the $j$ th UIR in the reduction of $V(\cdot)$ and $m$ performs a similar function in the reduction of $\widetilde{V}(\cdot)$.

We now regard the sets of $N_{j}^{2}$ states $\{|j m n\rangle\}$ for each fixed $j$ as momentum eigenstates in the present context. This means that the $n$-dimensional real momentum eigenvalue $\underline{p}$ in Cartesian quantum mechanics is now replaced by a collection of (discrete) quantum numbers $j \mathrm{jmn}$. A vector $|\psi\rangle \in \mathcal{H}$ with wave function $\psi(g)$ is given in the momentum description by a set of expansion coefficients $\psi_{j m n}$,

$$
\begin{gathered}
\psi \in \mathcal{H} \rightarrow \psi_{j m n}=\langle j m n \mid \psi\rangle=N_{j}^{1 / 2} \int_{G} \mathrm{~d} g D_{m n}^{j}(g) * \psi(g), \\
\|\psi\|^{2}=\sum_{j m n}\left|\psi_{j m n}\right|^{2} .
\end{gathered}
$$

A normalized $|\psi\rangle$ then determines two complementary probability distributions, $|\psi(g)|^{2}$ on $G$ and $\left|\psi_{j m n}\right|^{2}$ on momentum space.

In this situation a (provisional and overcomplete) Wigner distribution $\widetilde{W}\left(g ; j m n m^{\prime} n^{\prime}\right)$ can be defined for each $|\psi\rangle \in \mathcal{H}$ (or for any mixed state $\hat{\rho}$ as well). (Here we depart slightly from the 
notation in Ref. 21, so that our later expressions are more concise.) It transforms in a reasonable manner when $|\psi\rangle$ is acted upon by $V(\cdot)$ or $\tilde{V}(\cdot)$; and it reproduces in a simple and direct way the two probability distributions determined by $|\psi\rangle$, as marginals. We give only the latter property here,

$$
\begin{gathered}
\sum_{j m n} \tilde{W}(g ; j m n m n)=|\psi(g)|^{2}, \\
\int_{G} \mathrm{~d} g \tilde{W}\left(g ; j m n m^{\prime} n^{\prime}\right)=\psi_{j m^{\prime} n^{\prime}} \psi_{j m n}^{*} .
\end{gathered}
$$

The right-hand side of the second relation is a natural generalization of $\left|\psi_{j m n}\right|^{2}$, to allow for freedom in the choice of labels $m, n$ within each UIR $j$. The expression for this Wigner distribution involves a function $s, G \times G \rightarrow G$ obeying certain conditions and is

$$
\tilde{W}\left(g ; j m n m^{\prime} n^{\prime}\right)=N_{j} \int_{G} \mathrm{~d} g^{\prime} \int_{G} \mathrm{~d} g^{\prime \prime} \psi\left(g^{\prime \prime}\right) \psi\left(g^{\prime}\right) * D_{m^{\prime} n^{\prime}}^{j}\left(g^{\prime \prime}\right) * D_{m n}^{j}\left(g^{\prime}\right) \delta\left(g^{-1} s\left(g^{\prime}, g^{\prime \prime}\right)\right) \text {. }
$$

Reality in the Cartesian or single angle-angular momentum cases is replaced here by Hermiticity,

$$
\tilde{W}\left(g ; j m n m^{\prime} n^{\prime}\right) *=\tilde{W}\left(g ; j m^{\prime} n^{\prime} m n\right) .
$$

The conditions on $s\left(g^{\prime}, g^{\prime \prime}\right)$ to ensure that all the above properties are secured are

$$
\begin{gathered}
g^{\prime}, g^{\prime \prime} \in G \rightarrow s\left(g^{\prime}, g^{\prime \prime}\right)=s\left(g^{\prime \prime}, g^{\prime}\right) \in G, \\
s\left(g_{1} g^{\prime} g_{2}, g_{1} g^{\prime \prime} g_{2}\right)=g_{1} s\left(g^{\prime}, g^{\prime \prime}\right) g_{2}, \\
s\left(g^{\prime}, g^{\prime}\right)=g^{\prime} .
\end{gathered}
$$

We can simplify the problem of constructing such a function by exploiting the second of these relations to write

$$
s\left(g^{\prime}, g^{\prime \prime}\right)=g^{\prime} s\left(e, g^{\prime-1} g^{\prime \prime}\right)=g^{\prime} s_{0}\left(g^{\prime-1} g^{\prime \prime}\right),
$$

so the function $s_{0}(g)$ of a single group element must satisfy

$$
\begin{gathered}
s_{0}(e)=e, \\
s_{0}\left(g^{-1}\right)=g^{-1} s_{0}(g)=s_{0}(g) g^{-1}, \\
s_{0}\left(g^{\prime} g g^{\prime-1}\right)=g^{\prime} s_{0}(g) g^{\prime-1} .
\end{gathered}
$$

The solution proposed in Ref. 21 is to take $s_{0}(g)$ to be the midpoint along the geodesic from the identity $e \in G$ to $g$. These geodesics are determined starting from the invariant Cartan-Killing metric on $G$, and have the necessary behaviors under left and right group actions to ensure that all of Eqs. (3.26) and (3.28) are obeyed. In the exponential notation of Eq. (3.8) we have

$$
s_{0}\left(\exp \left(\alpha^{r} e_{r}\right)\right)=\exp \left(\frac{1}{2} \alpha^{r} e_{r}\right)
$$

since it is true that geodesics passing through the identity are one parameter subgroups. With this explicit construction we have the additional relation 


$$
s_{0}\left(g^{-1}\right)=s_{0}(g)^{-1}
$$

i.e.,

$$
s_{0}(g) s_{0}(g)=g .
$$

Thus $s_{0}(g)$ is the (almost everywhere unique) square root of $g$ and $s\left(g^{\prime}, g^{\prime \prime}\right)$ is a kind of symmetric square root of $g^{\prime}$ and $g^{\prime \prime}$.

We shall explore the properties of $\widetilde{W}\left(g ; j m n m^{\prime} n^{\prime}\right)$ in the next section, especially the sense in which it contains information about $|\psi\rangle\langle\psi|$ in an overcomplete manner. This will then lead to the Wigner-Weyl isomorphism for quantum mechanics on a (compact simple) Lie group.

\section{THE WIGNER-WEYL ISOMORPHISM IN THE LIE GROUP CASE}

The definition (3.24) can be immediately extended to associate an object $\widetilde{W}_{\hat{A}}\left(g ; j m n m^{\prime} n^{\prime}\right)$ with every linear operator $\hat{A}$ on $\mathcal{H}$ (of Hilbert-Schmidt class). In terms of the integral kernel $\left\langle g^{\prime \prime}|\hat{A}| g^{\prime}\right\rangle$ of $\hat{A}$ we have

$$
\widetilde{W}_{\hat{A}}\left(g ; j m n m^{\prime} n^{\prime}\right)=N_{j} \int_{G} \mathrm{~d} g^{\prime} \int_{G} \mathrm{~d} g^{\prime \prime}\left\langle g^{\prime \prime}|\hat{A}| g^{\prime}\right\rangle D_{m^{\prime} n^{\prime}}^{j}\left(g^{\prime \prime}\right) * D_{m n}^{j}\left(g^{\prime}\right) \delta\left(g^{-1} s\left(g^{\prime}, g^{\prime \prime}\right)\right) .
$$

It is indeed the case that this expression describes or determines $\hat{A}$ completely, however this happens in an overcomplete manner. There are certain linear relations obeyed by $\widetilde{W}_{\hat{A}}\left(g ; j m n m^{\prime} n^{\prime}\right)$ which have an $\hat{A}$ independent form. We now obtain these relations, then proceed to construct a simpler expression which contains complete information about $\hat{A}$ without redundancy.

The Dirac delta function in the integral on the right-hand side of Eq. (4.1) means that the only contributions to the integral are from the points where

$$
s\left(g^{\prime}, g^{\prime \prime}\right)=g .
$$

Writing this as

$$
s_{0}\left(g^{\prime-1} g^{\prime \prime}\right)=g^{\prime-1} g,
$$

and then using Eq. (3.30), we see that, say, in the $g^{\prime \prime}$ integration the delta function picks out the single point determined by

$$
g^{\prime-1} g^{\prime \prime}=\left(g^{\prime-1} g\right)^{2}
$$

i.e.,

$$
g^{\prime \prime}=g g^{\prime-1} g
$$

This means that $\delta\left(g^{-1} s\left(g^{\prime}, g^{\prime \prime}\right)\right)$ is some Jacobian factor times $\delta\left(g^{\prime \prime-1} g g^{\prime-1} g\right)$. We are therefore permitted to use this value for $g^{\prime \prime}$ elsewhere in the integrand, so

$$
\widetilde{W}_{\hat{A}}\left(g ; j m n m^{\prime} n^{\prime}\right)=N_{j} \int_{G} \mathrm{~d} g^{\prime} \int_{G} \mathrm{~d} g^{\prime \prime}\left\langle g g^{\prime-1} g|\hat{A}| g^{\prime}\right\rangle D_{m^{\prime} n^{\prime}}^{j}\left(g g^{\prime-1} g\right) * D_{m n}^{j}\left(g^{\prime}\right) \delta\left(g^{-1} s\left(g^{\prime}, g^{\prime \prime}\right)\right) .
$$

Transferring the $g$-dependent representation matrices from the right-hand side to the left-hand side and using unitarity, we get 


$$
\begin{aligned}
\sum_{m^{\prime} n^{\prime}} D_{m^{\prime} m^{\prime \prime}}^{j}(g) D_{n^{\prime \prime} n^{\prime}}^{j}(g) \tilde{W}_{\hat{A}}\left(g ; j m n m^{\prime} n^{\prime}\right)= & N_{j} \int_{G} \mathrm{~d} g^{\prime} \int_{G} \mathrm{~d} g^{\prime \prime} \delta\left(g^{-1} s\left(g^{\prime}, g^{\prime \prime}\right)\right) \\
& \times\left\langle g g^{\prime-1} g|\hat{A}| g^{\prime}\right\rangle D_{n^{\prime \prime} m^{\prime \prime}}^{j}\left(g^{\prime}\right) D_{m n}^{j}\left(g^{\prime}\right) .
\end{aligned}
$$

It is now clear we have symmetry of the expression on the left-hand side under the simultaneous interchanges $m \leftrightarrow n^{\prime \prime}, n \leftrightarrow m^{\prime \prime}$, a statement independent of $\hat{A}$. This is the sense in which $\tilde{W}_{\hat{A}}\left(g ; j m n m^{\prime} n^{\prime}\right)$ contains information about $\hat{A}$ in an overcomplete manner, and this happens only when $G$ is non-Abelian.

Taking advantage of this, we now associate to $\hat{A}$ the simpler quantity

$$
W_{\hat{A}}\left(g ; j m m^{\prime}\right)=N_{j}^{-1} \sum_{n} \tilde{W}_{\hat{A}}\left(g ; j m n m^{\prime} n\right)=\int_{G} \mathrm{~d} g^{\prime} \int_{G} \mathrm{~d} g^{\prime \prime}\left\langle g^{\prime \prime}|\hat{A}| g^{\prime}\right\rangle D_{m m^{\prime}}^{j}\left(g^{\prime} g^{\prime \prime-1}\right) \delta\left(g^{-1} s\left(g^{\prime}, g^{\prime \prime}\right)\right) .
$$

We shall call this the Weyl symbol corresponding to the operator $\hat{A}$. The passage $\hat{A} \rightarrow \hat{A}^{\dagger}$ results in

$$
W_{\hat{A}^{\dagger}}\left(g ; j m m^{\prime}\right)=W_{\hat{A}}\left(g ; j m^{\prime} m\right) *
$$

It is easy to obtain the transformation properties of the Weyl symbol under conjugation of $\hat{A}$ by either the left or the right regular representation,

$$
\begin{gathered}
\hat{A}^{\prime}=V\left(g_{0}\right) \hat{A} V\left(g_{0}\right)^{-1}, \\
W_{\hat{A}^{\prime}}\left(g ; j m m^{\prime}\right)=\sum_{m_{1} m_{1}^{\prime}} D_{m m_{1}}^{j}\left(g_{0}\right) D_{m^{\prime} m_{1}^{\prime}}^{j}\left(g_{0}\right) * W_{\hat{A}}\left(g_{0}^{-1} g ; j m_{1} m_{1}^{\prime}\right), \\
\hat{A}^{\prime \prime}=\widetilde{V}\left(g_{0}\right) \hat{A} \widetilde{V}\left(g_{0}\right)^{-1}, \\
W_{\hat{A}^{\prime \prime}}\left(g ; j m m^{\prime}\right)=W_{\hat{A}}\left(g g_{0} ; j m m^{\prime}\right) .
\end{gathered}
$$

Next we can verify that if $\hat{A}$ and $\hat{B}$ are any two Hilbert-Schmidt operators on $\mathcal{H}$, then $\operatorname{Tr}(\hat{A} \hat{B})$ can be simply expressed in terms of their Weyl symbols,

$$
\operatorname{Tr}(\hat{A} \hat{B})=\sum_{j m m^{\prime}} N_{j} \int_{G} \mathrm{~d} g W_{\hat{A}}\left(g ; j m m^{\prime}\right) W_{\hat{B}}\left(g ; j m^{\prime} m\right) .
$$

The proof exploits the completeness relation in (3.20) and the properties (3.26) of $s\left(g^{\prime}, g^{\prime \prime}\right)$. This key result proves that $\hat{A}$ is indeed completely determined by its Weyl symbol: $\hat{A}$ is certainly determined by the values of $\operatorname{Tr}(\hat{A} \hat{B})$ for all $\hat{B}$, and the latter are known once the Weyl symbols are known.

Before expressing the Weyl symbol of $\hat{A}$ in a form analogous to Eq. (2.16), we give examples for some simple choices of $\hat{A}$, 


$$
\begin{array}{cc}
\hat{A}=\int_{G} \mathrm{~d} g f(g)|g\rangle\langle g| & W_{\hat{A}}\left(g ; j m m^{\prime}\right) \\
V\left(g_{0}\right) & f(g) \delta_{m m^{\prime}} \\
\tilde{V}\left(g_{0}\right) & D_{m m^{\prime}}^{j}\left(g_{0}^{-1}\right) \\
\hat{f} V\left(g_{0}\right) & D_{m m^{\prime}}^{j}\left(g g_{0} g^{-1}\right) \\
V\left(g_{0}\right) \hat{f} & f\left(s_{0}\left(g_{0}\right) g\right) D_{m m^{\prime}}^{j}\left(g_{0}^{-1}\right) \\
& f\left(s_{0}\left(g_{0}\right)^{-1} g\right) D_{m m^{\prime}}^{j}\left(g_{0}^{-1}\right)
\end{array}
$$

We shall comment later on the structure of these Weyl symbols. However it is already instructive to compare these results with the Cartesian situation

$$
\begin{array}{cc}
\hat{A} & W(q, p) \\
\hline \hat{f}=f(\hat{q}) & f(q) \\
V\left(q^{\prime}\right) & \exp \left(-i p q^{\prime}\right) \\
\hat{f} V\left(q^{\prime}\right)=f(\hat{q}) V\left(q^{\prime}\right) & f\left(q+q^{\prime} / 2\right) \exp \left(-i p q^{\prime}\right) \\
V\left(q^{\prime}\right) f(\hat{q}) & f\left(q-q^{\prime} / 2\right) \exp \left(-i p q^{\prime}\right)
\end{array}
$$

Now we turn to the problem of expressing the Weyl symbol of $\hat{A}$ in the form

$$
W_{\hat{A}}\left(g ; j m m^{\prime}\right)=\operatorname{Tr}\left(\hat{A} \hat{W}\left(g ; j m m^{\prime}\right)\right)
$$

for a suitable operator $\hat{W}\left(g ; j m m^{\prime}\right)$. This would be the analogue of $\hat{W}(q, p)$ in Eq. (2.10). Since the kernel $\left\langle g^{\prime \prime}|\hat{A}| g^{\prime}\right\rangle$ is quite general, Eq. (4.13) and Eq. (4.7) imply

$$
\left\langle g^{\prime}\left|\hat{W}\left(g ; j m m^{\prime}\right)\right| g^{\prime \prime}\right\rangle=D_{m m^{\prime}}^{j}\left(g^{\prime} g^{\prime \prime-1}\right) \delta\left(g^{-1} s\left(g^{\prime}, g^{\prime \prime}\right)\right)=D_{m m^{\prime}}^{j}\left(g^{\prime} g^{\prime \prime-1}\right) \delta\left(g^{-1} s_{0}\left(g^{\prime \prime} g^{\prime-1}\right) g^{\prime}\right) .
$$

We shall synthesize $\hat{W}\left(g ; j m m^{\prime}\right)$ in steps. We begin by defining a family of commuting operators $U(j m n)$ in the manner of Eq. (3.5), all of them diagonal in the position basis,

$$
(U(j m n) \psi)(g)=D_{m n}^{j}(g) \psi(g) .
$$

These are analogous to the Cartesian $U\left(p^{\prime}\right)$, labeled by a momentum eigenvalue $j m n$, functions of position alone. They are unitary in the matrix sense,

$$
\sum_{m} U(j m n)^{\dagger} U\left(j m n^{\prime}\right)=\sum_{m} U(j n m)^{\dagger} U\left(j n^{\prime} m\right)=\delta_{n^{\prime} n} I .
$$

These operators allow us to express the map $f \in \mathcal{F}(G) \rightarrow \hat{f}$ of Eq. (3.5) more explicitly as follows:

$$
f(g)=\sum_{j m n} f_{j m n} D_{m n}^{j}(g) \Rightarrow \hat{f}=\sum_{j m n} f_{j m n} U(j m n) .
$$

Upon conjugation by $V(g)$ we have

$$
V(g)^{-1} U(j m n) V(g)=\sum_{m^{\prime}} D_{m m^{\prime}}^{j}(g) U\left(j m^{\prime} n\right) .
$$

Combining Eqs. (3.16), (3.20), and (4.15) we easily obtain the trace orthonormality property 


$$
\operatorname{Tr}\left(\left(U\left(j^{\prime} m^{\prime} n^{\prime}\right) V\left(g^{\prime}\right)\right)^{\dagger} U(j m n) V(g)\right)=N_{j}^{-1} \delta_{j^{\prime} j} \delta_{m^{\prime} m} \delta_{n^{\prime} n} \delta\left(g^{-1} g^{\prime}\right),
$$

analogous to Eqs. (2.7) and (2.5). The action of $U\left(j^{\prime} m^{\prime} n^{\prime}\right)$ on the momentum eigenstates $|j m n\rangle$ can be worked out; it involves the Clebsch-Gordan coefficients for the reduction of direct products of two general UIR's of $G$ and reads

$$
U\left(j^{\prime} m^{\prime} n^{\prime}\right)|j m n\rangle=\sum_{j^{\prime \prime} m^{\prime \prime} n^{\prime \prime} \lambda} \sqrt{\frac{N_{j}}{N_{j^{\prime \prime}}}} C_{m^{\prime} m j^{\prime} j^{\prime \prime} \lambda} * C_{n^{\prime} n n^{\prime \prime}}^{j^{\prime} j{ }^{\prime \prime} \lambda}\left|j^{\prime \prime} m^{\prime \prime} n^{\prime \prime}\right\rangle .
$$

Here $\lambda$ is a multiplicity index keeping track of the possibly multiple occurrences of the UIR $D^{j^{\prime \prime}}$ in the reduction of the direct product $D^{j^{\prime}} \times D^{j}$. The significance of this relation is similar in spirit to the statement in the Cartesian case that $U\left(p^{\prime}\right)=\exp \left(i p^{\prime} \hat{q}\right)$ generates a translation in $\hat{p}$, in other words that in the momentum description $\hat{q}$ is given by the differential operator $i(\mathrm{~d} / \mathrm{d} p)$. The result (4.20) however involves discrete labels since $G$ is compact, unlike continuous Cartesian variables, and incorporates non-Abelianness as well. Therefore translating the momentum jmn by the amount $j^{\prime} m^{\prime} n^{\prime}$ yields several final momenta $j^{\prime \prime} m^{\prime \prime} n^{\prime \prime}$ according to the contents of the direct product $D^{j^{\prime}} \times D^{j}$ of UIR's of $G$.

Now multiply both sides of Eq. (4.14) by $D_{m_{1} m_{1}^{\prime}}^{j_{1}}(g)$ and integrate with respect to $g$, this is Fourier transformation with respect to $g$ and gives

$$
\left\langle g^{\prime}\left|\int_{G} \mathrm{~d} g D_{m_{1} m_{1}^{\prime}}^{j_{1}}(g) \hat{W}\left(g ; j m m^{\prime}\right)\right| g^{\prime \prime}\right\rangle=D_{m m^{\prime}}^{j}\left(g^{\prime} g^{\prime \prime-1}\right) D_{m_{1} m_{1}^{\prime}}^{j_{1}}\left(s_{0}\left(g^{\prime \prime} g^{\prime-1}\right) g^{\prime}\right) .
$$

Now perform an inverse Fourier transformation with respect to the momenta $\mathrm{jmm}^{\prime}$ to get

$$
\begin{aligned}
& \sum_{j m m^{\prime}} N_{j} D_{m m^{\prime}}^{j}\left(g_{1}\right) *\left\langle g^{\prime}\left|\int_{G} \mathrm{~d} g D_{m_{1} m_{1}^{\prime}}^{j_{1}}(g) \hat{W}\left(g ; j m m^{\prime}\right)\right| g^{\prime \prime}\right\rangle \\
& \quad=D_{m_{1} m_{1}^{\prime}}^{j_{1}}\left(s_{0}\left(g^{\prime \prime} g^{\prime-1}\right) g^{\prime}\right) \delta\left(g_{1} g^{\prime \prime} g^{\prime-1}\right)=D_{m_{1} m_{1}^{\prime}}^{j_{1}}\left(s_{0}\left(g_{1}^{-1}\right) g^{\prime}\right) \delta\left(g_{1} g^{\prime \prime} g^{\prime-1}\right) \\
& =\left\langle g^{\prime} \mid g_{1} g^{\prime \prime}\right\rangle D_{m_{1} m_{1}^{\prime}}^{j_{1}}\left(s_{0}\left(g_{1}^{-1}\right) g^{\prime}\right)=\sum_{m_{2}}\left\langle g^{\prime}\left|U\left(j_{1} m_{2} m_{1}^{\prime}\right) V\left(g_{1}\right)\right| g^{\prime \prime}\right\rangle D_{m_{1} m_{2}}^{j_{1}}\left(s_{0}\left(g_{1}^{-1}\right)\right) .
\end{aligned}
$$

Comparing the two sides and peeling off $\left\langle g^{\prime}\right|$ and $\left|g^{\prime \prime}\right\rangle$ gives

$$
\sum_{j m m^{\prime}} N_{j} D_{m m^{\prime}}^{j}\left(g_{1}\right) * \int_{G} \mathrm{~d} g D_{m_{1} m_{1}^{\prime}}^{j_{1}}(g) \hat{W}\left(g ; j m m^{\prime}\right)=\sum_{m_{2}} D_{m_{1} m_{2}}^{j_{1}}\left(s_{0}\left(g_{1}^{-1}\right)\right) U\left(j_{1} m_{2} m_{1}^{\prime}\right) V\left(g_{1}\right) .
$$

Then Fourier inversion twice yields the result

$$
\hat{W}\left(g ; j m m^{\prime}\right)=\sum_{j_{1} m_{1} m_{2}} N_{j_{1}} \int_{G} \mathrm{~d} g_{1} U\left(j_{1} m_{2} m_{1}\right) V\left(g_{1}\right) D_{m m^{\prime}}^{j}\left(g_{1}\right) D_{m_{1} m_{2}}^{j_{1}}\left(g^{-1} s_{0}\left(g_{1}^{-1}\right)\right) .
$$

This may be compared in every detail with the Cartesian result in Eq. (2.10), the correspondence of arguments and integration/summation variables is (including the factors representing momentum eigenfunctions)

$$
\begin{gathered}
q \rightarrow g, \quad p \rightarrow j m m^{\prime}, \quad q^{\prime} \rightarrow g_{1}, \quad p^{\prime} \rightarrow j_{1} m_{1} m_{2}, \\
e^{i p q^{\prime}} \rightarrow D_{m m^{\prime}}^{j}\left(g_{1}\right), \quad e^{-i p^{\prime}\left(q+q^{\prime} / 2\right)} \rightarrow D_{m_{1} m_{2}}^{j_{1}}\left(g^{-1} s_{0}\left(g_{1}^{-1}\right)\right) .
\end{gathered}
$$

Giving due attention to the new matrix features, the correspondence is quite remarkable.

Combining Eqs. (3.28) and (4.14) we obtain the relation 


$$
\hat{W}(g ; j m n)^{\dagger}=\hat{W}(g ; j n m) .
$$

Similarly combining Eqs. (4.24) and (4.19) and carrying out quite elementary operations leads to analogues to the Cartesian relations (2.11) and (2.15) in the forms

$$
\begin{gathered}
\operatorname{Tr}\left(\hat{W}\left(g^{\prime} ; j^{\prime} m^{\prime} n^{\prime}\right)^{\dagger} \hat{W}(g ; j m n)\right)=N_{j}^{-1} \delta_{j j^{\prime}} \delta_{m m^{\prime}} \delta_{n n^{\prime}} \delta\left(g^{-1} g^{\prime}\right), \\
\hat{A}=\sum_{j m n} N_{j} \int_{G} \mathrm{~d} g W_{\hat{A}}(g ; j n m) \hat{W}(g ; j m n) .
\end{gathered}
$$

We may thus conclude that we have succeeded in setting up a Wigner-Weyl isomorphism for quantum mechanics on a compact simple Lie group with reasonable properties.

\section{THE STAR PRODUCT FOR WEYL SYMBOLS}

In this section we sketch the derivation of the expression for noncommutative operator multiplication in terms of the corresponding Weyl symbols, relegating some details to the Appendix. Thus, for two operators $\hat{A}$ and $\hat{B}$ we seek an expression for the Weyl symbol of $\hat{A} \hat{B}$ in terms of those of $\hat{A}$ and $\hat{B}$ in the form

$$
W_{\hat{A} \hat{B}}(g ; j m n)=\left(W_{\hat{A}} \star W_{\hat{B}}\right)(g ; j m n) .
$$

From Eq. (4.13) we have

$$
\left(W_{\hat{A}} \star W_{\hat{B}}\right)(g ; j m n)=\operatorname{Tr}(\hat{A} \hat{B} \hat{W}(g ; j m n)),
$$

so using Eq. (4.27) for $\hat{A}$ as well as for $\hat{B}$ we have

$$
\begin{aligned}
\left(W_{\hat{A}} \star W_{\hat{B}}\right)(g ; j m n)= & \sum_{j^{\prime} m^{\prime} n^{\prime}} N_{j^{\prime}} N_{j^{\prime \prime}} \int_{G} \mathrm{~d} g^{\prime \prime} \int_{G} \mathrm{~d} g^{\prime} W_{\hat{A}}\left(g^{\prime \prime} ; j^{\prime \prime} n^{\prime \prime} m^{\prime \prime}\right) W_{\hat{B}}\left(g^{\prime} ; j^{\prime} n^{\prime} m^{\prime}\right) \\
& j^{\prime \prime} m^{\prime \prime} n^{\prime \prime} \\
& \times \operatorname{Tr}\left(\hat{W}\left(g^{\prime \prime} ; j^{\prime \prime} m^{\prime \prime} n^{\prime \prime}\right) \hat{W}\left(g^{\prime} ; j^{\prime} m^{\prime} n^{\prime}\right) \hat{W}(g ; j m n)\right) .
\end{aligned}
$$

We therefore need to compute the trace of the product of three $\hat{W}$ 's, which is a nonlocal integral kernel defining the (associative but noncommutative) star product on the left-hand side. The two ingredients for this calculation are expressions for the product $U(j m n) V(g)$ in terms of $\hat{W}\left(g^{\prime} ; j^{\prime} m^{\prime} n^{\prime}\right)$, and for the product $U\left(j^{\prime} m^{\prime} n^{\prime}\right) V\left(g^{\prime}\right) U(j m n) V(g)$ in terms of similar products $U V$. These are

$$
\begin{aligned}
& U(j m n) V(g)=\sum_{j^{\prime} m^{\prime} n^{\prime}} N_{j^{\prime}} D_{m^{\prime} n^{\prime}}^{j^{\prime}}(g) * \int_{G} \mathrm{~d} g^{\prime} D_{m n}^{j}\left(s_{0}(g) g^{\prime}\right) \hat{W}\left(g^{\prime} ; j^{\prime} m^{\prime} n^{\prime}\right), \\
& U\left(j^{\prime} m^{\prime} n^{\prime}\right) V\left(g^{\prime}\right) U(j m n) V(g)=\sum_{j^{\prime \prime} m^{\prime \prime} n^{\prime \prime} k} C_{m^{\prime} n^{\prime}}^{j^{\prime}} \underset{k n}{j} \stackrel{j^{\prime \prime} m^{\prime \prime} n^{\prime \prime}}{\prime \prime} D_{m k}^{j}\left(g^{\prime-1}\right) U\left(j^{\prime \prime} m^{\prime \prime} n^{\prime \prime}\right) V\left(g^{\prime} g\right) .
\end{aligned}
$$

The derivations are given in the Appendix, and the $C$-symbol on the right-hand side in the second equation is a sum of products of Clebsch-Gordan coefficients of the type occurring in Eq. (4.20).

Starting from Eq. (4.24) and using Eq. (5.4b) we have for the product of two $\hat{W}$ 's, 


$$
\begin{aligned}
\hat{W}\left(g^{\prime} ; j^{\prime} m^{\prime} n^{\prime}\right) \hat{W}(g ; j m n) & =\sum_{\substack{j_{0}, m_{0}, n_{0} \\
j_{0}^{\prime} m_{0}^{\prime} n_{0}^{\prime}}} N_{j_{0}} N_{j_{0}^{\prime}} \int_{G} \mathrm{~d} g_{0} \int_{G} \mathrm{~d} g_{0}^{\prime} D_{m n}^{j}\left(g_{0}\right) D_{m^{\prime} n^{\prime}}^{j^{\prime}}\left(g_{0}^{\prime}\right) \\
& \times D_{n_{0} m_{0}}^{j_{0}}\left(g^{-1} s_{0}\left(g_{0}^{-1}\right)\right) D_{n_{0}^{\prime} m_{0}^{\prime}}^{j_{0}^{\prime}}\left(g^{\prime-1} s_{0}\left(g_{0}^{\prime-1}\right)\right) U\left(j_{0}^{\prime} m_{0}^{\prime} n_{0}^{\prime}\right) V\left(g_{0}^{\prime}\right) U\left(j_{0} m_{0} n_{0}\right) V\left(g_{0}\right) \\
= & \sum_{\substack{j_{0} m_{0} n_{0} k_{0} \\
j_{0}^{\prime} m_{0}^{\prime} n_{0}^{\prime}}} N_{j_{0}} N_{j_{0}^{\prime}} C_{m_{0}^{\prime} n_{0}^{\prime} k_{0} n_{0}}^{j_{j}^{\prime \prime}} m_{0}^{\prime \prime \prime} n_{0}^{\prime \prime} \int_{G} \mathrm{~d} g_{0} \int_{G} \mathrm{~d} g_{0}^{\prime} D_{m n}^{j}\left(g_{0}\right) D_{m^{\prime} n^{\prime}}^{j^{\prime}}\left(g_{0}^{\prime}\right) \\
& \times D_{m_{0} n_{0}}^{j_{0} k_{0}}\left(g_{0}^{\prime-1}\right) D_{n_{0} m_{0}}^{j_{0}}\left(g^{-1} s_{0}\left(g_{0}^{-1}\right)\right) D_{n_{0}^{\prime} m_{0}^{\prime}}^{j_{0}^{\prime}}\left(g^{\prime-1} s_{0}\left(g_{0}^{\prime-1}\right)\right) \times U\left(j_{0}^{\prime \prime} m_{0}^{\prime \prime} n_{0}^{\prime \prime}\right) V\left(g_{0}^{\prime} g_{0}\right) .
\end{aligned}
$$

If here we use Eq. (5.4a) and then Eq. (4.27) we obtain for the kernel in Eq. (5.3),

$$
\begin{aligned}
& \operatorname{Tr}\left(\hat{W}\left(g^{\prime \prime} ; j^{\prime \prime} m^{\prime \prime} n^{\prime \prime}\right) \hat{W}\left(g^{\prime} ; j^{\prime} m^{\prime} n^{\prime}\right) \hat{W}(g ; j m n)\right) \\
& =\sum_{\substack{j_{0} m_{0} n_{0} k_{0} \\
j_{0}^{\prime} m_{0}^{\prime} n_{0}^{\prime} \\
j_{0}^{\prime \prime} m_{0}^{\prime \prime} n_{0}^{\prime \prime}}} N_{j_{0}} N_{j_{0}^{\prime}} C_{m_{0}^{\prime} n_{0}^{\prime} k_{0}^{\prime} k_{0} j_{0}}^{j_{0}^{\prime \prime}} m_{0}^{\prime \prime} n_{0}^{\prime \prime} \int_{G} \mathrm{~d} g_{0} \int_{G} \mathrm{~d} g_{0}^{\prime} D_{m n}^{j}\left(g_{0}\right) D_{m^{\prime} n^{\prime}}^{j^{\prime}}\left(g_{0}^{\prime}\right) D_{n^{\prime \prime} m^{\prime \prime}}^{j^{\prime \prime}}\left(g_{0}^{\prime} g_{0}\right) * \\
& \quad \times D_{m_{0} k_{0}}^{j_{0}}\left(g_{0}^{\prime-1}\right) D_{n_{0} m_{0}}^{j_{0}}\left(g^{-1} s_{0}\left(g_{0}^{-1}\right)\right) D_{n_{0}^{\prime} m_{0}^{\prime}}^{j_{0}^{\prime}}\left(g^{\prime-1} s_{0}\left(g_{0}^{\prime-1}\right)\right) D_{m_{0}^{\prime \prime} n_{0}^{\prime \prime}}^{j_{0}^{\prime \prime}}\left(s_{0}\left(g_{0}^{\prime} g_{0}\right) g^{\prime \prime}\right) .
\end{aligned}
$$

The star product of Eq. (5.3) is then obtained by inserting this integral kernel on the right-hand side.

A slightly simpler expression-which amounts to trading four of the $D$-functions for Dirac delta functions-results from direct use of Eq. (4.14),

$$
\begin{aligned}
\operatorname{Tr}\left(\hat{W}\left(g^{\prime \prime} ; j^{\prime \prime} m^{\prime \prime} n^{\prime \prime}\right) \hat{W}\left(g^{\prime} ; j^{\prime} m^{\prime} n^{\prime}\right) \hat{W}(g ; j m n)\right) \\
=\int_{G} \mathrm{~d} g_{0} \int_{G} \mathrm{~d} g_{0}^{\prime} \int_{G} \mathrm{~d} g_{0}^{\prime \prime}\left\langle g_{0}\left|\hat{W}\left(g^{\prime \prime} ; j^{\prime \prime} m^{\prime \prime} n^{\prime \prime}\right)\right| g_{0}^{\prime}\right\rangle\left\langle g_{0}^{\prime}\left|\hat{W}\left(g^{\prime} ; j^{\prime} m^{\prime} n^{\prime}\right)\right| g_{0}^{\prime \prime}\right\rangle\left\langle g_{0}^{\prime \prime}|\hat{W}(g ; j m n)| g_{0}\right\rangle \\
=\int_{G} \mathrm{~d} g_{0} \int_{G} \mathrm{~d} g_{0}^{\prime} \int_{G} \mathrm{~d} g_{0}^{\prime \prime} D_{m^{\prime \prime} n^{\prime \prime}}^{j^{\prime \prime}}\left(g_{0} g_{0}^{\prime-1}\right) D_{m^{\prime} n^{\prime}}^{j^{\prime}}\left(g_{0}^{\prime} g_{0}^{\prime \prime-1}\right) \\
\quad \times D_{m n}^{j}\left(g_{0}^{\prime \prime} g_{0}^{-1}\right) \delta\left(g^{\prime \prime-1} s\left(g_{0}, g_{0}^{\prime}\right)\right) \delta\left(g^{\prime-1} s\left(g_{0}^{\prime}, g_{0}^{\prime \prime}\right)\right) \delta\left(g^{-1} s\left(g_{0}^{\prime \prime}, g_{0}\right)\right)
\end{aligned}
$$

These expressions for the star product show an unavoidable complexity for general compact non-Abelian $G$. In the one-dimensional Abelian (but non-Cartesian) case $Q=\mathrm{S}^{1}$, there are some simplifications. Referring to Sec. II, we have the rule for Weyl symbols given by Eq. (2.25) and (2.28),

$$
\begin{gathered}
a(\theta ; m)=\operatorname{Tr}(\hat{A} \hat{W}(\theta ; m)), \\
\hat{A}=2 \pi \sum_{m \in \mathbb{Z}} \int_{-\pi}^{\pi} \mathrm{d} \theta a(\theta ; m) \hat{W}(\theta ; m) .
\end{gathered}
$$

The star product then appears as 


$$
\begin{gathered}
(a \star b)(\theta ; m)=\sum_{m^{\prime}, m^{\prime \prime} \in Z} \int_{-\pi}^{\pi} \mathrm{d} \theta^{\prime \prime} \int_{-\pi}^{\pi} \mathrm{d} \theta^{\prime} \operatorname{Tr}\left(\hat{W}\left(\theta^{\prime \prime} ; m^{\prime \prime}\right) \hat{W}\left(\theta^{\prime} ; m^{\prime}\right) \hat{W}(\theta ; m)\right) a\left(\theta^{\prime \prime} ; m^{\prime \prime}\right) b\left(\theta^{\prime} ; m^{\prime}\right) \\
\begin{aligned}
\operatorname{Tr}\left(\hat{W}\left(\theta^{\prime \prime} ; m^{\prime \prime}\right) \hat{W}\left(\theta^{\prime} ; m^{\prime}\right) \hat{W}(\theta ; m)\right)= & \frac{1}{4 \pi^{2}} \sum_{m_{0}, m_{0}^{\prime} \in Z} \int_{-\pi}^{\pi} d \theta_{0}^{\prime} \int_{-\pi}^{\pi} d \theta_{0} e^{i / 2\left(m_{0}^{\prime} \theta_{0}-m_{0} \theta_{0}^{\prime}\right)} \exp \left[i \left(m \theta_{0}-m_{0} \theta\right.\right. \\
& \left.\left.+m^{\prime} \theta_{0}^{\prime}-m_{0}^{\prime} \theta^{\prime}+\left(m_{0}+m_{0}^{\prime}\right) \theta^{\prime \prime}-m^{\prime \prime}\left(\theta_{0}+\theta_{0}^{\prime}\right)\right)\right] .
\end{aligned}
\end{gathered}
$$

This expression for the kernel results from Eq. (5.6) if we first drop the magnetic quantum numbers $m, n, m^{\prime}, n^{\prime}, m^{\prime \prime}, n^{\prime \prime}, m_{0}, n_{0}, k_{0}, m_{0}^{\prime}, n_{0}^{\prime}, m_{0}^{\prime \prime}, n_{0}^{\prime \prime}$; then set the dimensionalities $N_{j 0}, N_{j_{0}^{\prime}}$ equal to unity; next make the replacements $j \rightarrow m, j^{\prime} \rightarrow m^{\prime}, j^{\prime \prime} \rightarrow m^{\prime \prime}, j_{0} \rightarrow m_{0}, j_{0}^{\prime} \rightarrow m_{0}^{\prime}, g_{0} \rightarrow \theta_{0}, g_{0}^{\prime} \rightarrow \theta_{0}^{\prime}$,

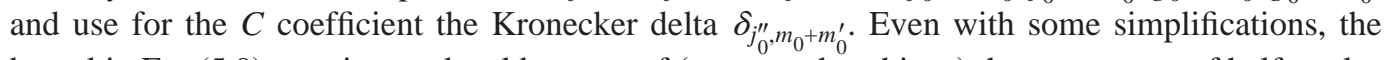
kernel in Eq. (5.9) remains nonlocal because of (among other things) the occurrence of half-angles in the exponent.

\section{DISCUSSION AND CONCLUDING REMARKS}

The characteristic feature revealed by our analysis is that for quantum mechanics on a Lie group $G$ as configuration space, the concept of canonical momentum is a collection of noncommuting operators $\hat{J}_{r}$, in fact constituting the Lie algebra of the left regular representation of $G$ on $L^{2}(G)$. This in itself is known, but it results in the analogues of momentum eigenvalue being a set of discrete labels jmn, and the single Cartesian momentum eigenvector $|p\rangle$ being replaced by a multidimensional set of vectors $\{|j m n\rangle\}$. Other consequences of this non-Abelianness should be noted. One needs to work with both overcomplete and with complete nonredundant Weyl symbols for general operators $\hat{A}$ : the former are useful for reproducing in a simple manner the two complementary marginal probability distributions associated with a pure or mixed quantum state from its Wigner distribution as shown in Eq. (3.23); while the latter lead to the Wigner-Weyl isomorphism in a reasonable manner.

It is interesting that the Weyl symbols $W_{\hat{A}}\left(g ; j m m^{\prime}\right)$ are not complex valued functions on the classical phase space $T^{*} G$. They may be more compactly viewed as follows. Whereas by the Peter-Weyl theorem the Hilbert space $\mathcal{H}=L^{2}(G)$ carries each UIR $\mathcal{D}^{(j)}(\cdot)$ of $G$ as often as its dimension $N_{j}$, the structure of Eq. (4.7) leads us to define a smaller Hilbert space $\mathcal{H}_{0}$ carrying each UIR of $G$ exactly once:

$$
\begin{gathered}
\mathcal{H}_{0}=\sum_{j} \oplus \mathcal{H}^{(j)}, \\
\left.\mathcal{H}^{(j)}=\operatorname{Sp}\{\mid j m)\right\}, \quad \operatorname{dim} \mathcal{H}^{(j)}=N_{j}, \\
\left(j^{\prime} m^{\prime} \mid j m\right)=\delta_{j^{\prime} j} \delta_{m^{\prime} m},
\end{gathered}
$$

with $\mathcal{H}^{(j)}$ carrying the UIR $\mathcal{D}^{(j)}(\cdot)$ of $G$. Then the Weyl symbol of a general operator $\hat{A}$, $W_{\hat{A}}\left(g ; j m^{\prime}\right)$, may be regarded as a function of $g \in G$ and an operator on $\mathcal{H}_{0}$. This is evident from the examples of Weyl symbols given in Eq. (4.11); in the Cartesian case in Eq. (4.12) such features are of course absent. This can be understood also from the following point of view. In the normal quantum description an operator $\hat{A}$ on $\mathcal{H}=L^{2}(G)$ can be given via its kernel $\left\langle g^{\prime \prime}|\hat{A}| g^{\prime}\right\rangle$, or via its complementary diagonal plus off-diagonal matrix elements $\left\langle j^{\prime} m^{\prime} n^{\prime}|\hat{A}| j m n\right\rangle$. If in the latter we trade half of the labels for a dependence on a group element $g$, we arrive at the Weyl symbol $W_{\hat{A}}\left(g ; j m^{\prime}\right)$ viewed as a block diagonal operator on $\mathcal{H}_{0}$ with simultaneously a dependence on $g$. Thus while the Wigner-Weyl isomorphism does not work directly with the true classical phase 
space $T^{*} G$, it seems to use what may be called a noncommutative cotangent space, standing somewhere between $T^{*} G$ and operators on $L^{2}(G)$.

Nevertheless the link to functions on the classical phase space $T^{*} G$ can be established, as we will see below.

We may use the phrase "semiquantized phase space" for the space on which the Weyl symbols $W_{\hat{A}}(g ; j m n)$ of operators $\hat{A}$ are defined. It is to be understood that this phrase includes the restriction that only ( $g$-dependent) block-diagonal operators on $\mathcal{H}_{0}$ are encountered. This may be viewed as a superselection rule. In detail, given an operator $\hat{A}$ on $\mathcal{H}=L^{2}(G)$, we associate with it the $g$-dependent block-diagonal operator,

$$
\left.\tilde{A}(g)=\sum_{j} \sum_{m, n} \sqrt{N_{j}} W_{\hat{A}}(g ; j m n) \mid j m\right)(j n \mid,
$$

acting on $\mathcal{H}_{0}$, and we then have the connection

$$
\operatorname{Tr}_{\mathcal{H}}(\hat{A} \hat{B})=\int_{G} \mathrm{~d} g \operatorname{Tr}_{\mathcal{H}_{0}}(\widetilde{A}(g) \widetilde{B}(g)) .
$$

The Weyl symbol $\widetilde{A}(g)$ is simpler than $\hat{A}$ both in that it acts on the much smaller Hilbert space $\mathcal{H}_{0}$, and in that it is block diagonal.

To finally establish the link to suitable functions on the classical phase space $T^{*} G$, we exploit both the fact that the representation of $G$ on $\mathcal{H}_{0}$ has a multiplicity-free reduction into UIR's, and the fact that $\widetilde{A}(g)$ is block diagonal. Let us denote the generators of $G$ on $\mathcal{H}_{0}$ by $\hat{J}_{r}^{(0)}, r$ $=1,2, \ldots, n$. The Weyl symbol $\widetilde{A}(g)$ may initially be written as the direct sum of symbols $\widetilde{A}_{j}(g)$ acting within each subspace $\mathcal{H}^{(j)}$ in $\mathcal{H}_{0}$,

$$
\begin{gathered}
\tilde{A}(g)=\sum_{j} \oplus \tilde{A}_{j}(g), \\
\left.\widetilde{A}_{j}(g)=\sum_{m, n} \sqrt{N_{j}} W_{\tilde{A}}(g ; j m n) \mid j m\right)(j n \mid .
\end{gathered}
$$

Next, using the irreducibility of $\left\{\hat{J}_{r}^{(0)}\right\}$ acting on $\mathcal{H}^{(j)}$, we can expand $\widetilde{A}_{j}(g)$ uniquely as a sum of symmetrized polynomials in $\hat{J}_{r}^{(0)}$,

$$
\begin{gathered}
\tilde{A}_{j}(g)=\sum_{N=0,1, \ldots} \sum_{r_{1}, r_{2}, \ldots, r_{N}} a_{r_{1} \ldots r_{N}}(g ; j)\left\{\hat{J}_{r_{1}}^{(0)} \hat{J}_{r_{2}}^{(0)} \cdots \hat{J}_{r_{N}}^{(0)}\right\}_{S}^{(j)}, \\
\left\{\hat{J}_{r_{1}}^{(0)} \hat{J}_{r_{2}}^{(0)} \ldots \hat{J}_{r_{N}}^{(0)}\right\}_{S}^{(j)}=\frac{1}{N !} \sum_{P \in S_{N}}\left(\hat{J}_{r_{P(1)}}^{(0)} \ldots \hat{J}_{r_{P(N)}}^{(0)}\right)^{(j)} .
\end{gathered}
$$

Here the upper limit of $N$ is determined by the UIR $D^{j} ; S_{N}$ is the permutation group on $N$ symbols; and the superscript $(j)$ denotes the restriction to $\mathcal{H}^{(j)}$. The coefficients $a_{r_{1}, \ldots, r_{N}}(g ; j)$ are $c$-number quantities symmetric in $r_{1}, \ldots, r_{N}$. If we now replace their $j$ dependences by dependences on the independent mutually commuting Casimir operators $\hat{\mathcal{C}}$ of $G$, themselves symmetric homogeneous polynomials in $\hat{J}_{r}^{(0)}$, we can use (6.5) in (6.4) and write

$$
\tilde{A}(g)=\sum_{N=0}^{\infty} \sum_{r_{1}, \ldots, r_{N}} a_{r_{1}, \ldots, r_{N}}(g ; \hat{\mathcal{C}})\left\{\hat{J}_{r_{1}}^{(0)} \cdots \hat{J}_{r_{N}}^{(0)}\right\}_{S} .
$$

This expression for the Weyl symbol $\widetilde{A}(g)$ of $\hat{A}$ can now be set into one-to-one correspondence with the classical phase space function 


$$
a(g ; J)=\sum_{N=0}^{\infty} \sum_{r_{1}, \ldots, r_{N}} a_{r_{1}, \ldots, r_{N}}(g ; \mathcal{C}) J_{r_{1}} \cdots J_{r_{N}},
$$

where the commuting classical variables $J_{r}$ are the canonical momentum coordinates of the classical phase space $T^{*} G,{ }^{21}$ while $\mathcal{C}$ are invariant (Casimir) homogeneous polynomials in them. Thus we have the two-stage sequence of correspondences

$$
\hat{A} \text { on } \mathcal{H}=L^{2}(G) \Leftrightarrow \widetilde{A}(g)=\text { block-diagonal operator on } \mathcal{H}_{0} \leftrightarrow a(g ; J) \in \mathcal{F}\left(T^{*} G\right) \text {. }
$$

The importance of the multiplicity-free nature of the representation of $G$ on $\mathcal{H}_{0}$, and the superselection rule, is evident. In contrast to the Cartesian case in Sec. II, the appearance of the semiquantized phase space as an intermediate step is to be noted. We hope to return to this aspect in a future publication.

\section{APPENDIX}

We indicate here the derivations of Eqs. (5.4a) and (5.4b). For Eq. (5.4a), we begin with Eq. (4.23) and use the unitarity of the $D$-matrices to shift the $D$-matrix on the right-hand side to the left-hand side. This immediately gives Eq. (5.4a). For Eq. (5.4b) we begin with the decomposition of the product of two U's; from Eq. (4.15), using Eq. (A29) in Ref. 21,

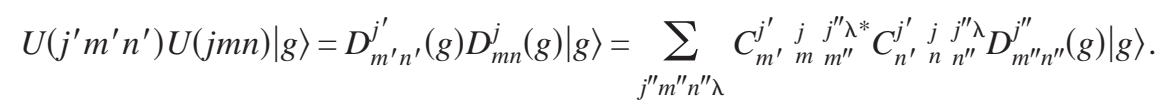

Here the $C$ 's are the usual Clebsch-Gordan coefficients for the decomposition of the direct product $D^{j^{\prime}} \times D^{j}$ of two UIR's into UIR's $D^{j^{\prime \prime}}$, with a multiplicity index $\lambda$ to keep track of multiple occurrences of a given $D^{j^{\prime \prime}}$. If we introduce the short-hand notation

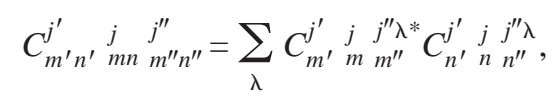

we get from (A1):

$$
U\left(j^{\prime} m^{\prime} n^{\prime}\right) U(j m n)=\sum_{j^{\prime \prime} m^{\prime \prime} n^{\prime \prime}} C_{m^{\prime} n^{\prime}}^{j^{\prime} m n} \underset{m^{\prime \prime} n^{\prime \prime}}{j^{\prime \prime}} U\left(j^{\prime \prime} m^{\prime \prime} n^{\prime \prime}\right)
$$

We can now tackle the product of four factors in Eq. (5.4b). First using Eqs. (3.7) and (4.18) and then using (A3) above gives

$$
\begin{aligned}
U\left(j^{\prime} m^{\prime} n^{\prime}\right) V\left(g^{\prime}\right) U(j m n) V(g) & =U\left(j^{\prime} m^{\prime} n^{\prime}\right) \sum_{k} D_{m k}^{j}\left(g^{\prime-1}\right) U(j k n) V\left(g^{\prime} g\right) \\
& =\sum_{j^{\prime \prime} m^{\prime \prime} n^{\prime \prime} k} D_{m k}^{j}\left(g^{\prime-1}\right) C_{m^{\prime} n^{\prime} \quad \begin{array}{l}
j^{\prime} \\
k n \\
j^{\prime \prime} \\
m^{\prime \prime} n^{\prime \prime}
\end{array}} U\left(j^{\prime \prime} m^{\prime \prime} n^{\prime \prime}\right) V\left(g^{\prime} g\right),
\end{aligned}
$$

which is Eq. (5.4b).

${ }^{1}$ E. P. Wigner, Phys. Rev. 40, 749 (1932); for a comprehensive review see M. Hillery, R. F. O’Connell, M. O. Scully, and E. P. Wigner, Phys. Rep. 106, 121 (1984); V. I. Tatarskii, Sov. Phys. Usp. 26, 311 (1983); and also Y. S. Kim and M. E. Noz, Phase Space Picture of Quantum Mechanics (World Scientific, Singapore, 1991); W. P. Schleich, Quantum Optics in Phase Space (Wiley-VCH, Weinheim, 2001).

${ }^{2}$ H. Weyl, Z. Phys. 46, 1 (1927); The Theory of Groups and Quantum Mechanics (Dover, New York, 1950).

${ }^{3}$ J. E. Moyal, Proc. Cambridge Philos. Soc. 45, 99 (1949); H. Groenewold, Physica (Amsterdam) 12, 405 (1946).

${ }^{4}$ For an elementary introduction to deformation quantization see A. C. Hirshfeld and P. Herselder, Am. J. Phys. 70, 537 (2002); J. Hancock, M. A. Walton, and B. Wynder, Eur. J. Phys. 25, 525 (2004).

${ }^{5}$ R. L. Stratonovich, Zh. Eksp. Teor. Fiz. 31, 1012 (1956) [Sov. Phys. JETP 4, 891 (1957)].

${ }^{6}$ G. S. Agarwal, Phys. Rev. A 24, 2889 (1981); 47, 4608 (1993); J. P. Dowling, G. S. Agarwal, and W. P. Schleich, ibid. 49, 4101 (1994)

${ }^{7}$ J. C. Várilly and J. M. Gracia-Bondía, Ann. Phys. (N.Y.) 190, 107 (1989). 
${ }^{8}$ K. B. Wolf, Opt. Commun. 132, 343 (1996).

${ }^{9}$ D. M. Kaplan and G. C. Summerfield, Phys. Rev. 187, 639 (1969).

${ }^{10}$ C. Fronsdal, Rep. Math. Phys. 15, 111 (1979).

${ }^{11}$ C. Moreno and P. Ortega-Navarro, Lett. Math. Phys. 7, 181 (1983).

${ }^{12}$ R. Gilmore, in Lecture Notes in Physics, Vol. 278, edited by Y. S. Kim and W. W. Zachary (Springer, Berlin, 1987), p. 211; W.-M. Zhang, D. H. Feng, and R. Gilmore, Rev. Mod. Phys. 62, 867 (1990).

${ }^{13}$ U. Leonhardt, Phys. Rev. Lett. 74, 4101 (1995); Phys. Rev. A 53, 2998 (1996).

${ }^{14}$ C. Brif and A. Mann, J. Phys. A 31, L9 (1998); Phys. Rev. A 59, 971 (1999).

${ }^{15}$ N. M. Atakishiyev, S. M. Chumakov, and K. B. Wolf, J. Math. Phys. 39, 6247 (1998); N. M. Nieto, N. M. Atakishiyev, S. M. Chumakov, and K. B. Wolf, J. Phys. A 16, 3875 (1998); S. T. Ali, N. M. Atakishiyev, S. M. Chumakov, and K. B. Wolf, Ann. Henri Poincare 1, 685 (2000); M. A. Alonso, G. S. Pogosyan, and K. B. Wolf, J. Math. Phys. 43, 5857 (2002).

${ }^{16}$ A. J. Bracken, D. Ellinas, and J. G. Wood, J. Phys. A 36, L297 (2003).

${ }^{17}$ N. Mukunda, Pramana 11, 1 (1978).

${ }^{18}$ N. Mukunda, Am. J. Phys. 47, 182 (1979).

${ }^{19}$ W. K. Wootters, Ann. Phys. (N.Y.) 176, 1 (1987).

${ }^{20}$ W. K. Wootters, IBM J. Res. Dev. 48, 99 (2003); quant-ph/0406032 (2004).

${ }^{21}$ N. Mukunda, Arvind, S. Chaturvedi, and R. Simon, J. Math. Phys. 45, 114 (2004).

${ }^{22}$ N. Mukunda, S. Chaturvedi, and R. Simon, Phys. Lett. A 321, 160 (2004). 